\title{
Modificaciones anatómicas de la derivación biliopancreática con cruce duodenal: ¿Tienen algún rol futuro en la cirugía bariátrica y metabólica?
}

\author{
Ramón Díaz J. ${ }^{1}$ Dana Portenier ${ }^{1}$, Rafael Luengas T. ${ }^{2}$ y A. Daniel Guerrón-Cruz ${ }^{1}$
}

\section{Anatomical modifications of the biliopancreatic diversion with duodenal switch: Do they have a future role in bariatric and metabolic surgery?}

Biliopancreatic Diversion with duodenal switch (BPD-DS) is the bariatric surgery that has shown the better results regarding long-term weight loss and comorbidities resolution. Nevertheless, BPD-DS' adoption has been slow, mainly due to its nutritional complications, and technical complexity. Given this, some authors have proposed surgical variations of this effective procedure. These new procedures are based on reducing the anastomosis to only one, and doing it just a loop anastomosis (no biliopacreatic diversion). These changes might bring to us the advantages of BPD-DS, and eliminate some of its disadvantages. In this article, we show the results of these variations compared with BPD-DS, and how their promising results could be a new approach for obese population and bariatric surgery.

Key words: biliopancreatic diversion; single anastomosis loop duodenal switch; morbid obesity.

\section{Resumen}

La derivación biliopancreática con cruce duodenal (BPD-DS) es el procedimiento bariátrico que ha mostrado los mejores resultados en cuanto a pérdida de peso y resolución de comorbilidades. Sin embargo, su adopción ha sido lenta, principalmente debido a sus complicaciones nutricionales y dificultad técnica. Dado esto, algunos autores han propuesto variaciones de este procedimiento. Estas están basadas en disminuir las anastomosis a solo una, y realizarla con un asa tipo loop (sin derivación biliopancreática). Estos cambios podrían reproducir las ventajas del BPD-DS, y eliminar algunas de sus desventajas. En este artículo, mostramos los resultados de estas variaciones comparadas con el BPD-DS, y cómo sus resultados prometedores pueden tener como consecuencia una nueva aproximación a la población que sufre de obesidad y sus comorbilidades

Palabras clave: derivación biliopancreática; cruce duodenal con anastomosis única en loop; obesidad mórbida.

\section{Introducción}

La derivación biliopancreática con cruce duodenal (BPD-DS) (Figura 1) es el procedimiento bariátrico que ha reportado los mejores resultados en cuanto a pérdida de peso y la resolución de comorbilidades. Sin embargo, la adopción de esta técnica quirúrgica no ha sido la esperada, ya sea por sus déficits nutricionales publicados o por su complejidad técnica ${ }^{1,2}$. En el afán de hacer llegar estos beneficios a una mayor población, algunos autores han publicado sus resultados con técnicas simplifi- cadas, manteniendo los beneficios metabólicos de la BPD-DS ${ }^{3}$. En este camino, los procedimientos con solo una anastomosis tipo loop, entre el duodeno y otro segmento del intestino delgado, han sido propuestos durante la última década. Como resultado de estas cirugías, en el asa loop, se genera un asa aferente (preanastomótica), y un asa eferente (posanastomótica). Estas variantes pueden ofrecer ventajas, tales como tiempos operatorios más cortos, menores pérdidas nutricionales y menores complicaciones relacionadas con la anastomosis; además, como ha sido demostrado por el grupo de
Weight Loss and Minimally Invasive Surgery Division, Duke University Health System, Durham N.C., United States of America.

${ }^{2}$ Clínica Red Salud Santiago, Santiago de Chile.

Recibido el 18 de octubre de 2018 y aceptado para publicación el 3 de diciembre de 2018.

Correspondencia a:

Dr. Ramón Díaz J.

ramon.diaz.jara@duke.edu 

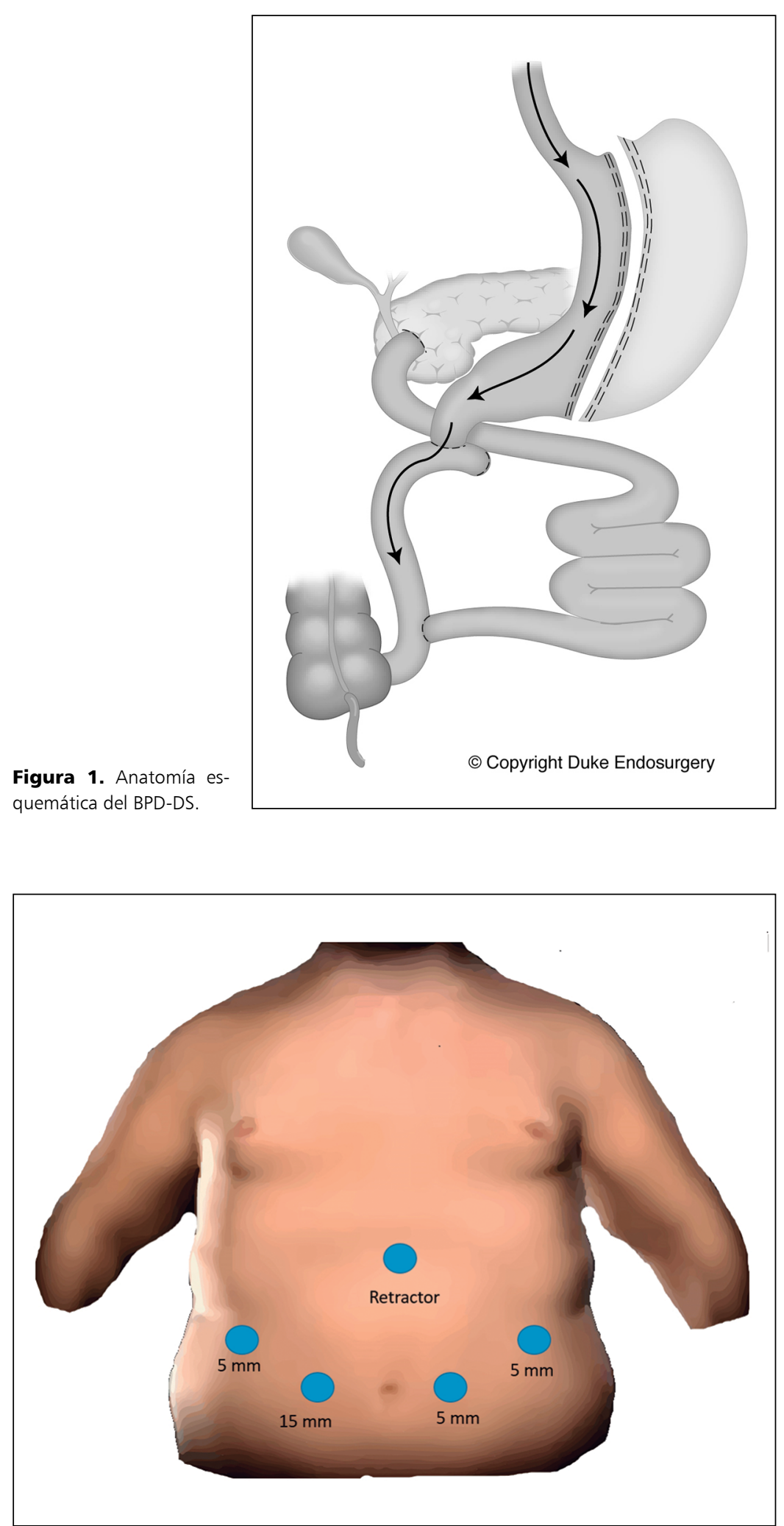

Figura 2. Esquema de la posición de los puertos de trabajo durante el SIPS, de izquierda a derecha miden: $5 \mathrm{~mm}, 15 \mathrm{~mm}, 5 \mathrm{~mm}, 5 \mathrm{~mm}$. En epigastrio el puerto de retracción mide $5 \mathrm{~mm}$.
DeMeester en estudios anteriores, al conservarse el píloro y algunos centímetros de duodeno, las posibilidades de síndrome de Dumping y úlceras de la boca anastomótica disminuyen en forma importante, lo que es importante para la calidad de vida posoperatoria en los pacientes sometidos a cirugía por obesidad ${ }^{4,5}$. Más aún, como el mesenterio de las asas intestinales no es seccionado, en teoría, no habría posibilidades de hernias internas, las que son una de las complicaciones más temidas en las reconstrucciones con Y-de-Roux ${ }^{6-8}$. No obstante, al subir el asa tipo loop se genera un espacio pseudo Petersen, por donde podría herniarse un segmento de intestino, pero hasta el momento no se han descrito en la literatura casos de hernia interna. El objetivo de este artículo es analizar y describir los resultados más importantes publicados hasta el momento en técnicas simplificadas del BPD-DS, y si estas técnicas tendrán un impacto en la práctica de la cirugía bariátrica. Describiremos principalmente los resultados de Single Anastomosis Duodeno-Ileal Bypass (SADI-S) y Stomach Intestinal PylorusSparing Surgery (SIPS), comparados con la BPDDS.

\section{Técnica quirúrgica}

Nuestro centro, actualmente, es uno de los que mayor volumen de BPD-DS tiene en Estados Unidos. Asimismo, también estamos familiarizados con la técnica del SIPS, la cual describimos brevemente a continuación:

Los trócares se posicionan como se muestra en la Figura 2. Luego de la separación hepática estándar o con un dispositivo magnético, se realiza una gastrectomía vertical tubular (GVT) calibrada con una sonda de 40-French. La sección gástrica se realiza con cargas de $60 \mathrm{~mm}$, las dos primeras de color negro y el resto moradas con material de refuerzo, partiendo aproximadamente a $5 \mathrm{~cm}$ del píloro. Posteriormente, se efectúa un túnel retrogástrico, desde el nivel donde comienza la sección del estómago hasta el nivel de la arteria gastroduodenal, aproximadamente $3 \mathrm{~cm}$ a distal al píloro. Este es un paso crítico, ya que se debe tener precaución en la disección para evitar un muñón duodenal isquémico. El duodeno se secciona con carga lineal de $60 \mathrm{~mm}$, igualmente con material de refuerzo. Se ejecuta la colecistectomía de rutina en estos pacientes. Como paso crítico, se cuenta desde la válvula ileocecal hacia retrógrado $300 \mathrm{~cm}$ de intestino delgado. Para confeccionar la anastomosis manual, se realiza una sutura corrida con sutura barbada absorbible 2-0 desde el borde antimesentérico al segmento duodenal que quedó como remanente y en conexión con 
el píloro. Efectuamos la duodenotomía y enterotomía, y se completa la anastomosis (cara posterior y cara anterior) con sutura multifilamento absorbible 2-0. Se comprueba hermeticidad con endoscopía, y cerramos la aponeurosis de los puertos (Figura 3). No realizamos prueba con otro medio como azul de metileno.

\section{Resultados}

Sánchez-Pernaute et al., publicaron por primera vez su técnica de SADI-S en el año $2007^{9}$. Este autor diseñó la GVT sobre una sonda de calibración de 54-French, y midió un asa eferente de 200 $\mathrm{cm}$ desde la válvula ileocecal. Posteriormente, los mismos autores describieron su experiencia con esta cirugía con 3 años de seguimiento ${ }^{10}$. En esta publicación, 50 pacientes fueron sometidos a cirugía sin complicaciones intraoperatorias, ni filtraciones de la duodeno-íleo anastomosis; durante ese seguimiento, los pacientes habían perdido el $100 \%$ de su exceso de peso (\%PEP), aunque $8 \%$ de los sujetos tuvo hipoalbuminemia en el mismo período. Cabe destacar que todos los pacientes necesitaron suplementación vitamínica, con calcio y vitamina $\mathrm{D}$, además $50 \%$ de ellos necesitó mayores dosis de lo habitual. En esta serie, en los primeros 15 casos, se realizó BILITEC 2000 (Medtronic Inc, Skovlunde, Denmark), sin hallar reflujo alcalino patológico ${ }^{10}$. Dada la malabsorción, el mismo grupo decidió alargar el asa eferente a $250 \mathrm{~cm}^{11}$. En esta nueva experiencia, los autores describieron la técnica con 100 pacientes $(50$ con $200 \mathrm{~cm} \mathrm{y}$ 50 con $250 \mathrm{~cm}$ de asa eferente), de los cuales 59 tenían diabetes mellitus tipo 2 (DM). Una limitante cuando se analizan los resultados de este estudio, es que los autores reportaron los resultados de ambas mediciones de asa eferente en forma combinada, lo que no nos permite distinguir si este alargamiento tiene beneficios nutricionales para los pacientes. No obstante, los dos pacientes que necesitaron revisión por hipoalbuminemia, fueron pacientes tratados con $200 \mathrm{~cm}$ de asa eferente. El seguimiento a los 4 años fue escaso, pero al año de efectuada la cirugía, casi todos los pacientes habían perdido la totalidad de su \%PEP, con un $92 \%$ de remisión de $\mathrm{DM}^{11}$. El mismo grupo publicó su experiencia en un estudio no randomizado, solo con 97 pacientes que padecían $\mathrm{DM}^{12}$. La remisión de la enfermedad fue $72 \%$ luego del primer año de cirugía; después de 5 años de seguimiento, la remisión alcanzó a un 52\%, siendo factores de riesgo negativo para remisión la duración de la enfermedad (7 años o más) o la

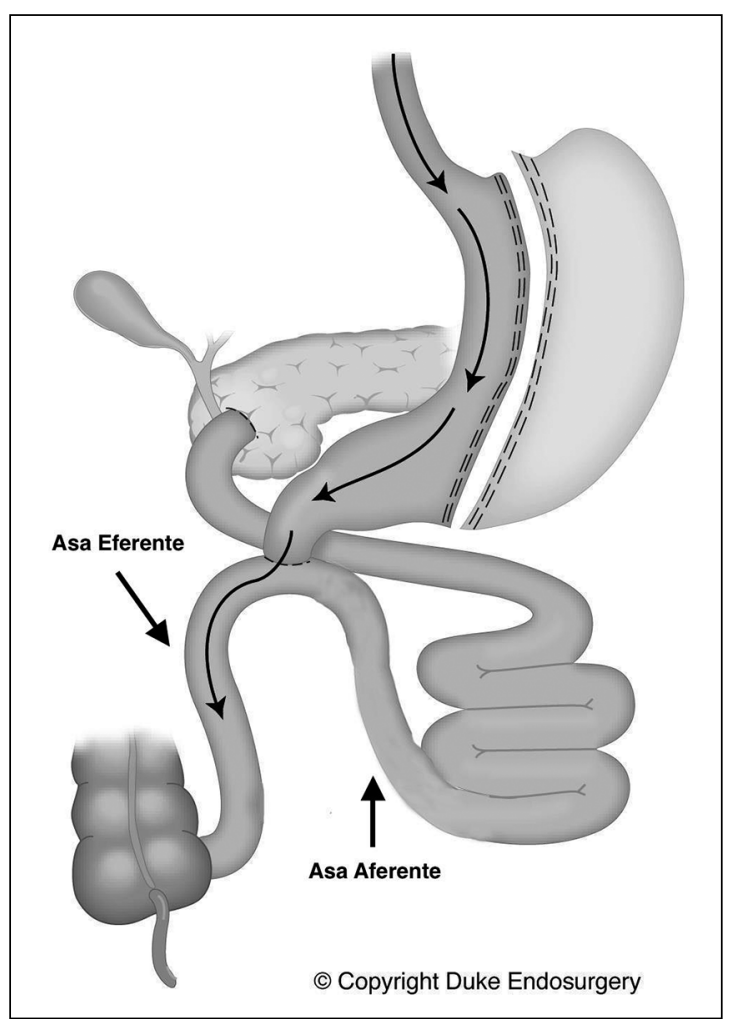

Figura 3. Anatomía es quemática del SIPS. Asa eferente mide $300 \mathrm{~cm}$, a diferencia del SADI-S donde mide $250 \mathrm{~cm}$. utilización de insulina previo a la cirugía ${ }^{12}$. Nelson et al, publicaron la primera experiencia fuera de Europa con 72 pacientes con SADI-S $(250 \mathrm{~cm}$ de asa eferente $)^{13}$. La pérdida de exceso de peso a los 12 meses fue $62 \%$; un total de 18 pacientes tenían DM, de los cuales la mitad remitió en idéntico período, el mismo fenómeno ocurrió con la hipertensión arterial que remitió en un $42 \%$ en ese seguimiento ${ }^{13}$. En una publicación donde se comparó el bypass gástrico laparoscópico (BGL), BPD-DS y SADI$\mathrm{S}^{14}$, la remisión de DM fue superior para SADI-S a $\operatorname{los} 3$ años de seguimiento, siendo 55,2, 70,4, y $75,8 \%$, respectivamente. De igual manera, en el mismo período de seguimiento la pérdida de peso es similar entre BPD-DS y SADI-S, y ambas son superiores al BGL ${ }^{14}$.

Mitzman et al, publicaron otra modificación del BPD-DS, ellos lo llamaron Stomach Intestinal Pylorus-Sparing (SIPS) ${ }^{15}$. Esta técnica es muy similar a SADI-S, pero se diferencia en que el asa eferente mide $300 \mathrm{~cm}$ versus $250 \mathrm{~cm}$ del SADI-S, y el tamaño de la calibración de la GVT es 42 versus 54-French del SADI-S. Los autores analizaron 123 pacientes, cuyo índice de masa corporal (IMC) promedio fue $49,4 \pm 9,2 \mathrm{Kg} / \mathrm{m}^{2}$. A los 12 meses, 
con un $62 \%$ de seguimiento, el $\%$ PEP promedio fue $72 \%$, y no se reportaron complicaciones relacionadas con la anastomosis duodeno-ileal o mortalidad $^{15}$. Recientemente Neichoy et al, describieron una serie con 225 pacientes a los cuales se le realizó $\mathrm{SIPS}^{16}$. El promedio de IMC preoperatorio fue 52,4 $\mathrm{Kg} / \mathrm{m}^{2}$, y el \%PEP a los 2 años de seguimiento fue $89 \%$. La remisión de DM fue $89 \%$, y los autores reportaron solo 5 filtraciones $(2,2 \%)$ de la anastomosis duodeno-ileal, y no reportaron síndrome de Dumping durante el seguimiento ${ }^{16}$. Cottam et al., realizaron un estudio comparativo entre SIPS versus BPD-DS ${ }^{17}$ con 61 pacientes por rama de estudio. Luego de 2 años de seguimiento, ninguna diferencia significativa se encontró en \%PEP y parámetros nutricionales. No hubo diferencia significativa entre las dos cirugías en términos de niveles normales de HbA1C $(<6,0)$; al año de seguimiento $86 \%$ de los pacientes con SIPS y $87 \%$ en el grupo de BPD-DS alcanzaron niveles normales. Además, el grupo en que se efectuó SIPS tuvo menores complicaciones a largo plazo, como diarrea ${ }^{17}$. SIPS igualmente ha exhibido buenos resultados cuando se le compara con $\mathrm{BGL}^{18}$. En un estudio no randomizado, SIPS tuvo mejor \%PEP, menores complicaciones posoperatorias, y mejor resolución de diabetes ${ }^{18}$. Los autores encontraron que en el $100 \%$ de los pacientes en la rama SIPS la DM remitió, comparado con el $75 \%$ en BGL, siendo estadísticamente significativo ${ }^{18}$. Surve et al, realizaron una comparación retrospectiva entre SIPS y BPD-DS ${ }^{19}$. SIPS tuvo menor tiempo operatorio, menor cantidad de filtraciones, menor diarrea a largo plazo, pero el \%PEP a los dos años de seguimiento fue significativamente mejor con BPD-DS (95 versus $87 \%$ ). Sin embargo, no hubo diferencias significativas entre estas dos cirugías cuando se compararon parámetros nutricionales, tales como vitamina D, B1, B12, calcio, albúmina sérica, proteínas séricas totales, y parámetros metabólicos como glicemia en ayuno, hemoglobina glicosilada, insulina basal, y perfil lipídico ${ }^{19}$. Recientemente Zaveri et al, publicaron una serie de 437 pacientes que se sometieron a SIPS $^{20}$. A los 4 años de seguimiento, los pacientes tuvieron un promedio de \%PEP de $86 \%$. Ocho pacientes desarrollaron complicaciones relacionadas con el largo del asa eferente ( 7 con diarrea, 1 hipoproteinemia), por lo tanto, se requirió alargamiento de dicho segmento del intestino delgado. La remisión de DM, dislipidemia, hipertensión arterial, y apnea del sueño, a los 4 años de seguimiento, fue $81,3,70,5,71$, y $64 \%$, respectivamente ${ }^{20}$.

\section{Conclusión}

Actualmente, la International Federation for the Surgery of Obesity and Metabolic Disorders (IFSO) recomienda llamar a ambos procedimientos como SADI-S ${ }^{21}$. Estas cirugías son interesantes ya que nos ofrecerían de manera simplificada los beneficios en pérdida de peso y metabólicos de la BPD-DS. Además, éstas resultan atractivas ya que eliminarían algunas de las complicaciones relacionadas con la anastomosis y, al no haber división del mesenterio, no habría hernias internas relacionadas con este defecto, pero como mencionamos existe un defecto por el cual podría migrar un asa de intestino, aunque no se ha descrito previamente. Asimismo, podría utilizarse como un recurso de cirugía de revisión luego de una GVT que presenta ganancia de peso, o como un second step en casos de pacientes de alto riesgo quirúrgico en quienes se realiza una GVT como primera aproximación ${ }^{22}$. Este último concepto es interesante debido a la gran cantidad de GVT que se realizan en la actualidad. La evidencia disponible hasta el momento es prometedora, pero debe ser analizada con criterio y mucha rigurosidad. Una mayor cantidad de estudios prospectivos con poder estadístico suficiente son necesarios para evaluar los resultados a largo plazo y complicaciones de estas propuestas. La American Society for Metabolic and Bariatric Surgery (ASMBS) publicó una declaración sobre estas variantes simplificadas del BPD-DS, donde las define como procedimientos en investigación, y que deben ser realizadas solo bajo protocolos de estudio estricto ${ }^{23}$. Como grupo donde realizamos unos de los mayores volúmenes de BPDDS en Estados Unidos, creemos que es importante e interesante buscar una simplificación de esta cirugía que pueda replicar los mismos resultados a largo plazo en cuanto a \%PEP y resolución de comorbilidades, sin las desventajas nutricionales. Sin embargo, el llamado es a que esta cirugía sea realizada solo en centro de alto volumen, y por cirujanos con experiencia en cirugía bariátrica.

\section{Responsabilidades éticas}

Protección de personas y animales. Los autores declaran que para esta investigación no se han realizado experimentos en seres humanos ni en animales.

Confidencialidad de los datos. Los autores declaran que en este artículo no aparecen datos de pacientes.

Conflictos de interés: no hay. 


\section{Referencias}

1. Sethi M, Chau E, Youn A, Jiang Y, Fielding G, Ren-Fielding C. Long-term outcomes after biliopancreatic diversion with and without duodenal switch: 2-, 5-, and 10-year data. Surg Obes Relat Dis. 2016;12:1697-705.

2. Skogar ML, Sundbom M. Duodenal Switch Is Superior to Gastric Bypass in Patients with Super Obesity when Evaluated with the Bariatric Analysis and Reporting Outcome System (BAROS). Obes Surg. 2017;27:2308-16.

3. Topart P, Becouarn G. The single anastomosis duodenal switch modifications: a review of the current literature on outcomes. Surg Obes Relat Dis. 2017;13:1306-12.

4. DeMeester TR, Fuchs KH, Ball CS, Albertucci M, Smyrk TC, Marcus JN. Experimental and clinical results with proximal end-to-end duodenojejunostomy for pathologic duodenogastric reflux. Ann Surg. 1987;206:414-26.

5. Welch NT, Yasui A, Kim CB, Barlow AP, Hinder RA, DeMeester TR, et al. Effect of duodenal switch procedure on gastric acid production, intragastric $\mathrm{pH}$, gastric emptying, and gastrointestinal hormones. Am J Surg. 1992;163:37-44; discussion-5.

6. Higa KD, Ho T, Boone KB. Internal hernias after laparoscopic Roux-en-Y gastric bypass: incidence, treatment and prevention. Obes Surg. 2003;13:350-4.

7. Comeau $\mathrm{E}$, Gagner $\mathrm{M}$, Inabnet $\mathrm{WB}$, Herron DM, Quinn TM, Pomp A. Symptomatic internal hernias after laparoscopic bariatric surgery. Surg Endosc. 2005;19:34-9.

8. Cho M, Pinto D, Carrodeguas L, Lascano C, Soto F, Whipple O, et al. Frequency and management of internal hernias after laparoscopic antecolic antegastric Rouxen-Y gastric bypass without division of the small bowel mesentery or closure of mesenteric defects: review of 1400 consecutive cases. Surg Obes Relat Dis. 2006;2:87-91.

9. Sánchez-Pernaute A, Rubio Herrera MA, Perez-Aguirre E, Garcia Perez JC,
Cabrerizo L, Diez Valladares L, et al. Proximal duodenal-ileal end-to-side bypass with sleeve gastrectomy: proposed technique. Obes Surg. 2007;17:1614-8

10. Sánchez-Pernaute A, Herrera MA, Perez-Aguirre ME, Talavera P, Cabrerizo L, Matia P, et al. Single anastomosis duodeno-ileal bypass with sleeve gastrectomy (SADI-S). One to three-year follow-up. Obes Surg. 2010;20:1720-6.

11. Sánchez-Pernaute A, Rubio MA, Pérez Aguirre E, Barabash A, Cabrerizo L, Torres A. Single-anastomosis duodenoileal bypass with sleeve gastrectomy: metabolic improvement and weight loss in first 100 patients. Surg Obes Relat Dis. 2013;9:731-5.

12. Sánchez-Pernaute A, Rubio MA, Cabrerizo L, Ramos-Levi A, PerezAguirre E, Torres A. Single-anastomosis duodenoileal bypass with sleeve gastrectomy (SADI-S) for obese diabetic patients. Surg Obes Relat Dis. 2015;11:1092-8.

13. Nelson L, Moon RC, Teixeira AF, Galvao M, Ramos A, Jawad MA. Safety and Effectiveness of Single Anastomosis Duodenal Switch Procedure: Preliminary Result from a Single Institution. Arq Bras Cir Dig. 2016;29 Suppl 1(Suppl 1):80-4.

14. Torres A, Rubio MA, Ramos-Levi AM, Sanchez-Pernaute A. Cardiovascular Risk Factors After Single Anastomosis Duodeno-Ileal Bypass with Sleeve Gastrectomy (SADI-S): a New Effective Therapeutic Approach? Curr Atheroscler Rep. 2017;19:58.

15. Mitzman B, Cottam D, Goriparthi R, Cottam S, Zaveri H, Surve A, et al. Stomach Intestinal Pylorus Sparing (SIPS) Surgery for Morbid Obesity: Retrospective Analyses of Our Preliminary Experience. Obes Surg. 2016;26:2098-104.

16. Neichoy BT, Schniederjan B, Cottam DR, Surve AK, Zaveri HM, Cottam A, et al. Stomach Intestinal Pylorus-Sparing Surgery for Morbid Obesity. JSLS 2018;22(1).
17. Cottam A, Cottam D, Portenier D, Zaveri H, Surve A, Cottam S, et al. A Matched Cohort Analysis of Stomach Intestinal Pylorus Saving (SIPS) Surgery Versus Biliopancreatic Diversion with Duodenal Switch with Two-Year Follow-up. Obes Surg. 2017;27:454-61.

18. Cottam A, Cottam D, Zaveri H, Cottam S, Surve A, Medlin W, et al. An Analysis of Mid-Term Complications, Weight Loss, and Type 2 Diabetes Resolution of Stomach Intestinal Pylorus-Sparing Surgery (SIPS) Versus Roux-En-Y Gastric Bypass (RYGB) with Three-Year FollowUp. Obes Surg. 2018;28:2894-902.

19. Surve A, Zaveri H, Cottam D, Belnap L, Cottam A, Cottam S. A retrospective comparison of biliopancreatic diversion with duodenal switch with single anastomosis duodenal switch (SIPSstomach intestinal pylorus sparing surgery) at a single institution with two year follow-up. Surg Obes Relat Dis. 2017;13:415-22.

20. Zaveri H, Surve A, Cottam D, Cottam A, Medlin W, Richards C, et al. Midterm 4-Year Outcomes with Single Anastomosis Duodenal-Ileal Bypass with Sleeve Gastrectomy Surgery at a Single US Center. Obes Surg. 2018;18:52. doi: 10.1186/s12893-018-0381-8.

21. Brown WA, Ooi G, Higa K, Himpens J, Torres A, SADI-S/OADS IF-atfrtlo. Single Anastomosis Duodenal-Ileal Bypass with Sleeve Gastrectomy/One Anastomosis Duodenal Switch (SADI-S/ OADS) IFSO Position Statement. Obes Surg. 2018;28:1207-16.

22. Balibrea JM, Vilallonga R, Hidalgo M, Ciudin A, González O, Caubet E, et al. Mid-Term Results and Responsiveness Predictors After Two-Step SingleAnastomosis Duodeno-Ileal Bypass with Sleeve Gastrectomy. Obes Surg. 2017;27:1302-8.

23. Kim J, American Society for M, Bariatric Surgery Clinical Issues C. American Society for Metabolic and Bariatric Surgery statement on single-anastomosis duodenal switch. Surg Obes Relat Dis. 2016;12:944-5. 